\title{
Ultraspecific and Highly Sensitive Nucleic Acid Detection by Integrating a DNA Catalytic Network with a Label-Free Microcavity
}

\author{
Dr. Yuqiang Wu, \\ Laboratory of Nanophotonics and Biosensing, Max Planck Insitute for the Science of Light, \\ Erlangen 91058, Germany \\ Dr. David Yu Zhang, \\ Department of Bioengineering Rice, University Houston, USA \\ Dr. Peng Yin, and \\ The Wyss Institute for Biologically Inspired Engineering and Department of Systems Biology, \\ Harvard Medical School, Boston, USA \\ Dr. Frank Vollmer \\ Laboratory of Nanophotonics and Biosensing, Max Planck Insitute for the Science of Light, \\ Erlangen 91058, Germany. Division of Biomedical Engineering, Brigham and Women's Hospital, \\ Harvard Medical School, Boston, USA \\ Yuqiang Wu: yuqiang.wu@mpl.mpg.de; David Yu Zhang: dyz1@rice.edu; Frank Vollmer: frank.vollmer@mpl.mpg.de
}

\begin{abstract}
Nucleic acid detection with label-free biosensors circumvents costly fluorophore functionalization steps associated with conventional assays by utilizing transducers of impressive ultimate detection limits. Despite this technological prowess, molecular recognition at a surface limits the biosensors' sensitivity, specificity, and reusability. It is therefore imperative to integrate novel molecular approaches with existing label-free transducers to overcome those limitations. Here, we demonstrate this concept by integrating a DNA strand displacement circuit with a micron-scale whispering gallery mode (WGM) microsphere biosensor. The integrated biosensor exhibits at least 25-fold improved nucleic acid sensitivity, and sets a new record for label-free microcavity biosensors by detecting $80 \mathrm{pM}$ ( $32 \mathrm{fmol}$ ) of a $22 \mathrm{nt}$ oligomer; this improvement results from the catalytic behavior of the circuit. Furthermore, the integrated sensor exhibits extremely high specificity; single nucleotide variants yield 40- to 100-fold lower signal. Finally, the same physical sensor was demonstrated to alternatingly detect 2 different nucleic acid sequences through 5 cycles of detection, showcasing both its reusability and its versatility.
\end{abstract}

(C) 2014 Wiley-VCH Verlag GmbH \& Co. KGaA, Weinheim

Correspondence to: Yuqiang Wu, yuqiang.wu@mpl.mpg. de; David Yu Zhang, dyz1@rice.edu; Frank Vollmer,

frank.vollmer@mpl.mpg.de.

Supporting Information

Supporting Information is available from the Wiley Online Library or from the author. 


\section{Introduction}

Because nucleic acids act to encode and regulate the expression of genes, sequence-specific detection of DNA and RNA is an important research and clinical goal. ${ }^{[1,2]}$ Conventional diagnostic technologies often use fluorescence-based assays to localize and quantitate nucleic acid molecules of interest, driving much of molecular biology. ${ }^{[3,4]}$ However, functionalizing oligonucleotides with fluorescent labels is typically a complex and expensive process that often skews physical and chemical properties, in turn affecting quantitative readout. ${ }^{[5]}$

Label-free technologies for detecting the concentration of nucleic acids, such as those based on plasmon resonance, ${ }^{[6,7]}$ electrochemical conductance,,${ }^{[2,8]}$ nanowires, ${ }^{[9]}$ mechanical resonance, ${ }^{[10]}$ micro-cantilevers, ${ }^{[11]}$ or optical whispering gallery mode (WGM) resonance, ${ }^{[12-17]}$ circumvent the need for fluorescence modifications; see Table 1 for a comparison. In particular, optical WGM biosensors are emerging as one of the most versatile and sensitive label-free biosensing technologies, providing various mechanisms for sensing, sizing, trapping, and manipulation. ${ }^{[12-22]}$ Although techniques based on nanowire and electrochemical transducers have achieved very high sensitivity for detecting DNA and RNA concentrations, WGM biosensors are simple to fabricate, can be easily functionalized as well as multiplexed, and are made from inexpensive optical fibers. ${ }^{[13,15,17,21,22]}$

Sequence-specific detection by direct DNA hybridization on WGM devices faces three important challenges: ${ }^{[2,13,22-26]}$ limited sensitivity, specificity, and reusability. First, years of work on advancing the device physics and engineering of WGM biosensors has improved the ultimate physical detection limits of WGM transducers. ${ }^{[12,16,19-21,27]}$ Although WGM biosensors have shown great sensitivity for protein detection, the specific detection of DNA has been limited to 1 nanomolar concentrations due to specificity and sensitivity limits set by hybridization. ${ }^{[22,24-26,28]}$ Novel molecular approaches are thus needed to overcome those limitations, mostly set by the inherent kinetics and thermodynamics of the process of molecular recognition through direct hybridization at the sensor surface. Second, noncognate sequences that differ slightly in sequence may bind nonspecifically to the functionalized device, generating false positive signals and preventing proper detection. Finally, different sensors must be constructed to detect different nucleic acid sequences.

DNA strand displacement techniques have recently emerged as a novel family of approaches to enzyme-free homogenous detection assays. ${ }^{[35-38]}$ Strand displacement circuits, for example, have been demonstrated to implement nucleic acid "catalysis" in which a nucleic acid sequence of interest effects the release of up to 100 nucleic acid molecules from metastable precursors; cascading such catalytic systems ${ }^{[38]}$ has shown overall turnover of about 1000. Recently, strand displacement has been engineered to allow ultraspecific hybridization assays with specificity approaching the theoretical limit based on thermodynamics. ${ }^{[35]}$ Although strand displacement technologies have significantly improved the specificity and sensitivity of homogeneous detection assays, the readout for this technology has previously been constrained to gel electrophoresis or fluorescence readout, neither of which is easily applicable to point-of-care or clinical diagnostics. ${ }^{[35,38-40]}$ 
Here we combine the advantages of WGM label-free readouts and DNA strand displacement circuits by constructing an integrated label-free biosensor. Our work features the capabilities of DNA nanotechnology combined with highly sensitive optical microcavities for breaking through previous performance limitations in nucleic acid detection. This integrated biosensor exhibits at least 25-fold improvement in molecular sensitivity over the conventional hybridization-based WGM nucleic acid sensor. The integrated biosensor is highly specific, able to distinguish single nucleotide polymorphisms (SNPs) via kinetics of mass loading/ unloading by a factor of 40 to 100 . Finally, we demonstrate sequential detection of two different targets through five cycles of repeated use of the same WGM sensor device by isothermal exchange of buffer solutions.

\section{System Design}

\subsection{WGM Biosensor}

WGM biosensors are micron scale optical cavities, such as glass microspheres, capable of confining light by total internal reflection in a small modal volume and only at specific resonance wavelengths. These tiny optical resonators exhibit ultra-narrow linewidth, associated with very high quality $\mathrm{Q}$ factor, and are extremely sensitive to the binding of biomolecules to the microcavity surface. The changes in permittivity upon binding of analyte result in a shift of resonance wavelength. The high $\mathrm{Q}$ factor enables the precise monitoring of small resonance wavelength shifts, a method known as the reactive biosensing principle, which is emerging as one of the most sensitive label-free microsystems biodetection mechanisms. ${ }^{[12]}$

Only a small number of components are needed for an experimental realization of a WGM biosensor (Figure 1a). In this implementation, a continuous-wave tunable distributed feedback (DFB) laser diode, operating in the telecom band at $\sim 1550 \mathrm{~nm}$ wavelength, excites a high Q WGM optical resonance in a silica microsphere via a tapered optical fiber. The taper is fabricated from single mode SMF-28 fiber using a microtorch to heat and at the same time pull apart the fiber. The microsphere is fabricated by melting the tip of a short piece of optical fiber so that surface tension in the melted glass tip forms a 300-400 $\mu \mathrm{m}$ in diameter silica microsphere. The microsphere-on-a-stem is then mounted on a microstage for controlled coupling to the tapered fiber region (Figure 1b), and after coupling, the fibercoupled microsphere is immersed in a $\sim 400 \mu \mathrm{L}$ o-ring droplet cell. For specific DNA detection, the microsphere is functionalized with biorecognition elements, here $\sim 22 \mathrm{mer}$ DNA oligonucleotides. The resonance wavelength shift of the sensor (Figure 1c), for example upon DNA hybridization, is quantitated in grams of nucleic acid mass loading per

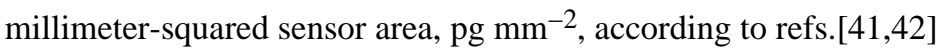

$$
\text { massloading }=\frac{\Delta \lambda}{\lambda} \frac{\left(n_{s}^{2}-n_{m}^{2}\right) R}{2 n_{m} \cdot d n / d c}
$$

where $\Delta \lambda$ is the shift of resonance wavelength, $\lambda$ is the nominal wavelength of the DFB laser, $n_{\mathrm{s}}=1.46$ and $n_{\mathrm{m}}=1.33$ are the refractive indices of microsphere and aqueous medium, respectively, $R$ is the approximate radius of the microsphere as determined by 
microscopic imaging, and $\mathrm{d} n / \mathrm{d} c \approx 0.17 \times 10^{-9}\left(\mathrm{~mm}^{3} \mathrm{pg}^{-1}\right)$ is the approximate incremental refractive index change of a DNA solution. ${ }^{[24]}$ For the DNA detection experiments presented in this work, single-stranded DNA oligonu-cleotide probes were attached via biotin-streptavidin linkers to a dextran hydrogel that was coated onto the silica micro-sphere by physisorption. ${ }^{[24]}$ The hybridization or dissociation of oligonucleotides with partial or full complementarity to these probes induces a mass change which can be observed from resonance wavelength shift, see Figure 1c and Experimental Section.

\subsection{Molecular amplification}

To improve the sensitivity of the WGM device at the molecular level, a "catalytic" DNA circuit based on strand displacement is designed, in which each molecule of the detection target effects the release of multiple molecules from multi-stranded precursor complexes. ${ }^{[38,43]}$ The two precursor molecules, F and SBP (Figure 2a), are designed based on the sequence of the detection target $\mathrm{C}$. In the absence of $\mathrm{C}, \mathrm{SBP}$ is double-stranded everywhere it is complementary to $\mathrm{F}$, so no significant reaction occurs, and the two species are metastable. $\mathrm{C}$ reacts with SBP to generate intermediate complex CBP and byproduct $\mathrm{S}$, the former of which can react with $\mathrm{F}$ via a newly exposed $5^{*}$ domain. At the end of a reaction cycle, $\mathrm{P}$ is released as a single-stranded product, and $\mathrm{C}$ is released to enable multiple turnover. Previous characterizations of similar catalysis systems ${ }^{[38,43]}$ reported catalytic speedup of over $10^{4}$ and maximum turnover of about 100. Polyacrylamide gel electrophoresis (PAGE) was used to verify that the specific sequences used for this integrated WGM system behave qualitatively similarly in bulk solution (see Figure S3).

To integrate the catalytic system with the WGM label-free sensor, product $\mathrm{P}$ is designed with a $3^{\prime}$ biotin modification, such that the SBP complex is initially functionalized to the glass microsphere via biotin-streptavidin interaction (Figure $2 b$ inset). In the presence of analyte $\mathrm{C}$, the catalytic reaction proceeds and $\mathrm{S}$ and $\mathrm{B}$ are released from $\mathrm{P}$ and the surface of the microsphere; this manifests as a surface mass unloading that yields a blue-shift of the resonance wavelength (Figure 1c). The integrated system should exhibit improved sensitivity to analyte $\mathrm{C}$, compared to a conventional hybridization assay, because each molecule of analyte $\mathrm{C}$ results in the release of multiple copies of $\mathrm{S}$ and $\mathrm{B}$ from the microsphere due to catalytic turnover.

\section{Results}

\subsection{Sensitivity Enhancement}

To experimentally characterize the sensitivity improvement afforded by the integration of the catalytic system, first the sensitivity of a standard hybridization-based WGM sensor for detecting a 22-mer oligonucleotide analyte $\mathrm{C}$ by direct hybridization to its complement $\mathrm{C}^{*}$ is determined. For this, the WGM biosensor surface is modified with complement $\mathrm{C}^{*}$ via biotin-streptavidin interactions (see Experimental Section). The microsphere sensor is immersed in the droplet cell filled with Tris-EDTA buffer for $\sim 10 \mathrm{~min}$ to allow temperature equilibration. Subsequently, a baseline sensogram was measured for $\sim 200 \mathrm{~s}$ before analyte $\mathrm{C}$ was injected at various concentrations ranging from 50 to $2 \mathrm{nM}$. The mass loading response for three independent measurements are shown in Figure 3a. With $50 \mathrm{nM}$ of $\mathrm{C}$, microsphere 
surface was saturated after $\sim 20$ minutes of reaction, whereas for lower concentrations, the hybridization reaction continued to occur past $\sim 40$ minutes.

In the $\sim 40$ minutes during which the mass loading was observed, the detection limit for $\mathrm{C}$ is approximately $2 \mathrm{nM}$. These results in terms of sensor response and timescales are consistent with previous DNA detection schemes using label-free WGM biosensors and direct hybridization $^{[24,26,28,44]}$ (see also Figure S9). Because the number of $C^{*}$ molecules functionalized to the surface of the microsphere is far fewer than the number of $\mathrm{C}$ molecules in solution (even at $2 \mathrm{nM} \mathrm{C}$, there is $10 \times$ excess of C; see Supporting Infomation), we infer that the hybridization reaction is kinetically limited, potentially by diffusion. Thus, in principle, significantly lower concentrations of $\mathrm{C}$ could be detected given enough reaction time - however, extended assay times are not conducive for many biomedical applications.

Next the sensitivity of the integrated system with a DNA catalytic network is determined where in the presence of analyte $\mathrm{C}, \mathrm{S}$ and $\mathrm{B}$ are released from $\mathrm{P}$ and the surface of the microsphere; this manifests as a surface mass unloading that yields a blue-shift of the resonance wavelength. Figure $3 \mathrm{~b}$ shows the experimental results in which $\mathrm{C}$ catalytically unloads molecules from the microsphere surface. The microsphere surface is initially loaded with pre-annealed SBP complex. At $\mathrm{t}=200 \mathrm{~s}, 4 \mu \mathrm{L}$ of strand $\mathrm{F}$ is injected to the $400 \mu \mathrm{L}$ droplet sample cell to a final concentration of $400 \mathrm{nM}$. At $\mathrm{t}=400 \mathrm{~s}$, analyte $\mathrm{C}$ is injected at concentrations of $50 \mathrm{nM}$ (red), $10 \mathrm{nM}$ (blue) $2 \mathrm{nM}$ (magenta), $400 \mathrm{pM}$ (green) or $80 \mathrm{pM}$ (orange). The control trace (black) shows the behavior of the system in the absence of $\mathrm{C}$.

To compare sensitivity at low concentrations the absolute initial slope of the mass loading (unloading) is used for quantification, a method established in WGM biosensing $[14,22,25]$ (Figure $3 \mathrm{c}$ ). With the integrated system, $80 \mathrm{pM}$ concentration of analyte $\mathrm{C}$ produces a mass unloading initial slope of $-0.17 \mathrm{pg} \mathrm{mm}^{-2} \mathrm{~s}$ (already corrected for the baseline slope from control experiment without target of $-0.031 \mathrm{pg} \mathrm{mm}^{-2} \mathrm{~s}$ ). For the conventional WGM sensor based on direct hybridization, $2 \mathrm{nM}$ concentration of analyte $\mathrm{C}$ produces a mass loading initial slope of $0.076 \mathrm{pg} \mathrm{mm}^{-2} \mathrm{~s}$, also corrected for the initial slope of $0.025 \mathrm{pg} \mathrm{mm}^{-2} \mathrm{~s}$ for the control experiments. Consequently, we have experimentally shown reliable detection of $\sim 80$ pM for 22-mer oligonucleotides, corresponding to at least 25-fold sensitivity improvement over the conventional WGM biosensor based on direct hybridization. At 80 pM concentration, there is less than 32 fmol of the DNA analyte in the droplet cell, setting a new sensitivity record for label-free DNA microcavity biosensors. ${ }^{[12,22]}$

Note that the total mass loading for the direct hybridization of target $\mathrm{C}$ is $\sim 1200 \mathrm{pg} / \mathrm{mm}^{2}$ (Figure 3a), while the total mass unloading for the WGM catalytic network mechanism is $\sim 3500 \mathrm{pg} \mathrm{mm}^{-2}$ (Figure 3b). This shows that the WGM signal is indeed proportional to the length of oligonucleotides: the loaded sequence $\mathrm{C}$ is $22 \mathrm{nt}$ long, and the unloaded sequences $\mathrm{S}$ and $\mathrm{B}$ are 20 and $49 \mathrm{nt}$ long, respectively. This also confirms that the WGM biosensor can be reproducibly modified with biotin-streptavidin linked oligonucleotides at surface concentrations of $\sim 10^{13}$ strands $\mathrm{cm}^{-2}$, consistent with previous observations for the dextran surface functionalization technique. ${ }^{[24]}$ 
To determine the turnover of this integrated system an alternative version of $\mathrm{F}$ that does not allow multiple turnover was designed. In these experiments (Figure S4), the kinetics of mass unloading is slowed significantly. From these measurements we infer that for $2 \mathrm{nM}$ of analyte $\mathrm{C}$, the averaged the turnover is $\sim 10$ after $40 \mathrm{~min}$ (Supporting Information).

We have also challenged our integrated sensor device with the task of detecting DNA oligonucleotides in a complex media for which we chose TE buffer with $10 \%$ fetal calf serum (FCS). Although the amplitude of our sensor response was reduced due to nonspecific bindings of FCS (Figure S5), our results show that our sensor is indeed able to detect target oligonucleotides with concentrations as low as $80 \mathrm{pM}$ - even in a complex media (Figure S6).

\subsection{Ultraspecificity and Single Nucleotide Discrimination}

Typically, label-free sensors based on hybridization struggles with single base specificity and discrimination of closely related nucleic acid analytes, due to the thermodynamic favorability of hybridization of non-cognate analytes with highly similar sequences. Although specificity for any particular nucleic acid analyte/probe pair can be optimized by solution salinity and temperature, ${ }^{[25]}$ this process is time consuming and imperfect, and not conducive to significant multiplexing. Similarly, the suppression of nonspecific interactions is essential to multiplexed detection.

Strand displacement circuits have overcome many of these challenges, and the integrated WGM sensor should in principle inherit the ultraspecificity properties of strand displacement systems. However, surface chemistry is known to deviate significantly from solution chemistry in both thermodynamics and kinetics, so it is necessary for us to experimentally test the integrated sensor's specificity.

To provide a benchmark for comparison, the standard hybridization-based WGM sensor was tested. We challenged this sensor with 3 single nucleotide variants of the intended analyte $\mathrm{C}$ (Cm5aG, $\mathrm{Cm} 11 \mathrm{cT}$, and $\mathrm{Cm} 14 \mathrm{gC}$; Figure $4 \mathrm{a}$ ). The three single base changes (A to $\mathrm{G}$ at position 5, $\mathrm{C}$ to $\mathrm{T}$ at position $11, \mathrm{G}$ to $\mathrm{C}$ at position 14), were selected to be representative of the variety of both positions along the analyte sequence and of the thermodynamics of single-base changes. These single nucleotide variants induced a similar kinetics and total amount of mass loading as the analyte C, so the standard WGM sensor is not specific to SNPs.

Figure $4 \mathrm{~b}$ shows the integrated strand displacement WGM sensor challenged by the same single nucleotide variants. Here, the single nucleotide variants showed significantly lower mass unloading than that of the intended analyte C. Quantitatively, a linear fit determined the initial slopes ${ }^{[22]}$ of the unloading to be $-14.5,-0.4,-0.13$ and $-0.1 \mathrm{pg} \mathrm{mm}^{-2} \mathrm{~s}$ for 50 $\mathrm{nM} \mathrm{C}, \mathrm{Cm} 5 \mathrm{aG}, \mathrm{Cm} 11 \mathrm{cT}$ and $\mathrm{Cm} 14 \mathrm{gC}$ respectively. From these unloading rates, we infer single-base specificity of the integrated biosensor to be between $40(-14.5 /-0.4)$ and 100 $(-14.5 /-0.1)$. The initial slight increase in mass loading may be due to the fact that the initial on-rates of the hybridization of some single nucleotide variants are higher than the unloading off-rates. This molecular specificity matches or exceeds the performance of strand 
displacement systems in bulk solution, ${ }^{[43]}$ and represents the first experimental demonstration of nucleic acid ultraspecificity at a surface.

\subsection{Versatility and Reusability}

Typical label-free nucleic acid detection technologies based on hybridization suffer from the limitation that a different functionalized device is needed to detect each different sequence. Furthermore, each device can generally be only used once; dehybridizing oligonucleotides requires harsh buffer conditions, high temperature, or practically takes too long. ${ }^{[2,45]}$ Here, the integrated sensor overcome both limitations: the same integrated WGM-strand displacement device can be reused to detect different sequences.

We first show that microspheres functionalized with the same DNA sequence $(\mathrm{P})$ could be used for the versatile detection of different analytes (Figure S7). This is feasible for our integrated system because the sequence of $\mathrm{P}$ is independent of the sequence of the detection target $C$. To detect a different target C2 (based on the vertebrate specific mir-194), a different set of sequences F2, S2, and B2, was designed which enables the same Pfunctionalized microsphere to detect $\mathrm{C} 2$.

The integrated system, in detecting the second analyte $\mathrm{C} 2$, exhibits significantly slower mass unloading than in detecting C. DNA folding software ${ }^{[46]}$ indicates that the F2 precursor possesses significant secondary structure. Thus, we believe that the kinetic slowdown is due to the kinetics of spontaneous unfolding of F2. ${ }^{[40]}$ In this particular case, the sequence of F2 is determined partially by the sequence of the common product $\mathrm{P}$, and partially by the sequence of the analyte $\mathrm{C} 2$. The sequence of $\mathrm{P}$, in turn, was designed specifically to avoid undesirable interactions with the first analyte $\mathrm{C}$, and thus resulted in unintended secondary structure of F2. Careful sequence design of $\mathrm{P}$ that considers all analytes should be able to overcome the kinetic slowdown due to secondary structure observed here for $\mathrm{C} 2$. Nonetheless, even with this non-ideal sequence setup, $\mathrm{C} 2$ is still reliably detected by the WGM strand-displacement sensor.

Next, the same WGM microsphere is shown to be reusable. At the end of a detection reaction, the products $\mathrm{S}$ and $\mathrm{FB}$ and the analyte $\mathrm{C}$ are released from the microsphere surface; consequently, through a mild buffer exchange and the WGM biosensor can be restored to allow repeated detection cycles (Figure S8). There is gradual decrease of maximum sensor signal (approximately $13 \%$ per cycle) upon repeated reuse of the same WGM device. The degradation of the sensor surface occurs when the functionalized microsphere is taken out of the droplet cell and partially dried in air. This degradation due to partial drying can be avoided in future devices integrated with microfluidics which will allow all steps of the detection cycle to be performed in solution.

Finally, we combine these two properties and show that the same WGM microsphere (functionalized with the same P molecules) can be used through 5 distinct cycles of detection to alternatingly detect 2 different sequences. The concept is demonstrated in Figure 5. 


\section{Conclusion}

For real-time, label-free nucleic acid detection, the integrated WGM biosensor provides three major advantages over conventional label-free biosensor approaches: molecular sensitivity, molecular specificity, and device reusability. Experimentally, we here showed at least 25 -fold enhancement of the sensitivity and detection down to $\sim 80 \mathrm{pM}(32 \mathrm{fmol}$ ) of a 22-mer DNA oligo both in clean buffer and FCS, SNP discrimination by a factor of 40 to 100 , and versatile detection by the same physical microsphere of 2 different analytes over 5 cycles of use.

The high sensitivity of the catalytic WGM strand-displacement is attributed to both the fact that the target DNA can catalytically trigger many detection events, and the fact that the mass unloading is greater than the size of the analyte oligonucleotide. The current catalyst system exhibits a turnover of about 10 in the course of the 40 minutes of reaction observed. Previous ${ }^{[43]}$ studies have shown that the catalytic turnover can be optimized to over 100 by adjustments such as the $5^{\prime} / 3^{\prime}$ orientation of molecules, the types of post-synthesis purifications, and the sequence design of the molecules. Furthermore, cascading two or more stages of catalysis ${ }^{[38]}$ allows asymptotically faster release kinetics and exponentially higher turnover. Additionally, because the mass of the analyte is decoupled from the mass being unloaded, the use of bulkier pre-loaded molecules ( $\mathrm{S}$ and $\mathrm{L}$ for this manuscript) can provide another easy way of significantly improving sensitivity. Consequently, the achievements shown in this manuscript are but the tip of an iceberg; significantly more sensitivity labelfree detection devices are on the horizon by further exploring the integration of strand displacement circuits with label-free biosensors.

The ultraspecificity displayed by the integrated WGM sensor is close to the theoretical maximum allowed by the thermodynamics of single base changes. ${ }^{[35]}$ Discrimination against single nucleotide variants of the intended analyte is robust to position and base identity of the SNP. Previous work has shown that in solution, these ultraspecific strand displacement systems retain their properties across a wide range of temperatures and salinities; although these properties were not tested here, we likewise believe that the integrated WGM biosensor can be robustly specific regardless of environmental conditions.

The demonstrations of versatility and reusability of the integrated WGM biosensor are important proofs-of-concept for transitioning this technology from the laboratory to realworld diagnostic applications. By allowing the same type of DNA-functionalized glass microspheres to be generally used for the detection of any arbitrary nucleic acid biomarker, device manufacturing costs are sharply reduced. By allowing the same physical microsphere device to be used across multiple cycles of operation, the number of devices needed by the end-users is reduced. Consequently, we are optimistic about the application of integrated WGM biosensors to point-of-care diagnostics.

\section{Experimental Section}

WGM biosensor setup-Silica microspherical WGM resonators with diameters ranging from 300 to $400 \mu \mathrm{m}$ were fabricated by melting the tip of optical fibers (SMF-28) with an 
oxygen-butane microtorch. The WGMs are excited by evanescent coupling the microsphere to a tapered optical fiber made by the standard heat-and-pull technique. The wavelength of a tunable distributed feedback laser (DFB) operating $\sim 1550 \mathrm{~nm}$ is scanned every millisecond to obtain a transmission spectrum of WGMs with a spectral width of $\sim 0.2 \mathrm{~nm}$. The spectra are recorded using LabVIEW program. The sample cell was prepared from an o-ring glued onto a glass slide. Inside our sample cell we place a miniature magnetic stir bar to homogenize the reactions. The schematic of the setup is shown in Figure 1a.

Microsphere surface modification-Microspheres were cleaned in an air oxygen plasma for 5 minutes immediately after fabrication, then immersed in a $2 \mu \mathrm{L}$ hanging drop of a dextran-biotin solution (10 mg/ml, Life Technologies) until almost dry. ${ }^{[24]}$ After a brief rinse in water for $5 \mathrm{~min}$, they are then incubated until almost dry in a hanging drop of $2 \mu \mathrm{L}$ of $8 \mu \mathrm{M}$ solution of biotinylated DNA oligonucleotides ( $\mathrm{C}^{*}$ or P), coupled to streptavidin at a molar ratio of $\sim 2: 1$. After incubation, spheres are rinsed again in the water and stored there until use. The dextran hydrogel coating is ideal for functionalizing the WGM biosensor since it prevents unspecific binding of DNA and supports a high surface density of attached biotin molecules that link to biotinlyated oligonucleotides via streptavidin. We have optimized the surface chemistry of dextran physisorption and repeatedly achieve a surface density of $\sim 10^{13} / \mathrm{cm}^{2}$ for biotin-streptavidin-linked DNA oligonucleotides (see Figure S1 \& S2).

DNA oligonucleotides and buffer conditions-The DNA oligonucleotides used in this study were purchased from Experience Eurofins MWG Operon. DNA oligonucleotides were purified by Eurofins using high-performance liquid chromatography (HPLC). Individual DNA oligonucleotides were re-suspended and stored in Tris-EDTA (TE) buffer (10 mM Tris-HCl pH balanced to 8.0, with $1 \mathrm{mM}$ disodium EDTA, purchased from SigmaAldrich) at $4{ }^{\circ} \mathrm{C}$. Directly preceding experiments, TE buffer is mixed with a final $\mathrm{MgCl}_{2}$ concentration of $12.5 \mathrm{mM}$. Because $\sim 1 \mathrm{mM}$ of the $\mathrm{Mg}^{2+}$ is chelated by the EDTA present in solution, the free concentration of $\mathrm{Mg}^{2+}$ is estimated to be $11.5 \mathrm{mM}$. All DNA sequences are listed in Table 2 and Table S1. Fetal calf serum was purchased from Biochrom, Merck Millipore.

\section{Supplementary Material}

Refer to Web version on PubMed Central for supplementary material.

\section{Acknowledgments}

Frank Vollmer acknowledges support from the Max Planck Society, Germany, as well as NIH grant 1R01GM095906-01 from the National Institutes of General Medical Sciences (NIGMS). DYZ acknowledges support from NIH grant EB015331 from the National Institute for Biomedical Imaging and Bioengineering (NIBIB). YW, DYZ, PY, and FV conceived the methods. YW and DYZ performed experiments. YW, DYZ, PY, and FV analyzed the data and wrote the manuscript.

\section{References}

1. Bartel DP. Cell. 2009; 136:215. [PubMed: 19167326]

2. Sassolas A, Leca-Bouvier BD, Blum LJ. Chem Rev. 2008; 108:109. [PubMed: 18095717] 
3. Tyagi S. Nat Methods. 2009; 6:331. [PubMed: 19404252]

4. Liti G, Carter DM, Moses AM, Warringer J, Parts L, James SA, Davey RP, Roberts IN, Burt A, Koufopanou V, Tsai IJ, Bergman CM, Bensasson D, O/'Kelly MJT, van Oudenaarden A, Barton DBH, Bailes E, Nguyen AN, Jones M, Quail MA, Goodhead I, Sims S, Smith F, Blomberg A, Durbin R, Louis EJ. Nature. 2009; 458:337. [PubMed: 19212322]

5. Marras SAE, Kramer FR, Tyagi S. Nucleic Acids Res. 2002; 30

6. Anker JN, Hall WP, Lyandres O, Shah NC, Zhao J, Van Duyne RP. Nat Mater. 2008; 7:442. [PubMed: 18497851]

7. Homola J. Chem Rev. 2008; 108:462. [PubMed: 18229953]

8. Drummond TG, Hill MG, Barton JK. Nat Biotechnol. 2003; 21:1192-1199. [PubMed: 14520405]

9. Patolsky F, Zheng G, Lieber CM. Nanomedicine. 2006; 1:51-65. [PubMed: 17716209]

10. Arlett JL, Myers EB, Roukes ML. Nat Nanotechnol. 2011; 6:203. [PubMed: 21441911]

11. Waggoner PS, Craighead HG. Lab Chip. 2007; 7:1238. [PubMed: 17896006]

12. Vollmer F, Yang L. Nanophotonics. 2012; 1:267.

13. Fan XD, White IM, Shopova SI, Zhu HY, Suter JD, Sun YZ. Anal Chim Acta. 2008; 620:8. [PubMed: 18558119]

14. Qavi A, Washburn A, Byeon JY, Bailey R. Anal Bioanal Chem. 2009; 394:121. [PubMed: 19221722]

15. Hunt HK, Armani AM. Nanoscale. 2010; 2:1544. [PubMed: 20820687]

16. Vollmer F, Arnold S. Nat Methods. 2008; 5:591. [PubMed: 18587317]

17. Yoshie T, Tang L, Su SY. Sensors. 2011; 11:1972. [PubMed: 22319393]

18. Lin S, Crozier KB. Lab Chip. 2011; 11:4047. [PubMed: 22011760]

19. Lu T, Lee H, Chen T, Herchak S, Kim JH, Fraser SE, Flagan RC, Vahala K. Proc Natl Acad Sci USA. 2011; 108:5976. [PubMed: 21444782]

20. Lopez-Yglesias X, Gamba JM, Flagan RC. J Appl Phys. 2012; 111:84701.

21. Vollmer F, Arnold S, Keng D. Proc Natl Acad Sci. 2008; 105:20701. [PubMed: 19075225]

22. Qavi AJ, Bailey RC. Angew Chemie-International Ed. 2010; 49:4608.

23. Nakatani K. Chembiochem. 2004; 5:1623. [PubMed: 15532027]

24. Vollmer F, Arnold S, Braun D, Teraoka I, Libchaber A. Biophys J. 2003; 85:1974. [PubMed: 12944310]

25. Qavi AJ, Mysz TM, Bailey RC. Anal Chem. 2011; 83:6827. [PubMed: 21834517]

26. Suter JD, White IM, Zhu HY, Shi HD, Caldwell CW, Fan XD. Biosens Bioelectron. 2008; 23:1003. [PubMed: 18036809]

27. Zhu J, Ozdemir SK, Xiao YF, Li L, He L, Chen DR, Yang L. Nat Phot. 2010; 4:46.

28. Scheler O, Kindt JT, Qavi AJ, Kaplinski L, Glynn B, Barry T, Kurg A, Bailey RC. Biosens Bioelectron. 2012; 36:56. [PubMed: 22541813]

29. Willets KA, Van Duyne RP. Annu Rev Phys Chem, Annual Reviews Palo Alto. 2007:267.

30. Milkani E, Morais S, Lambert CR, McGimpsey WG. Biosens Bioelectron. 2010; 25:1217. [PubMed: 19819685]

31. Šípová H, Homola J. Anal Chim Acta. 2013; 773:9. [PubMed: 23561902]

32. Hahm J, Lieber CM. Nano Lett. 2004; 4:51.

33. Burg TP, Godin M, Knudsen SM, Shen W, Carlson G, Foster JS, Babcock K, Manalis SR. Nature. 2007; 446:1066. [PubMed: 17460669]

34. Huber F, Lang HP, Backmann N, Rimoldi D, Gerber C. Nat Nanotechnol. 2013; 8:125. [PubMed: 23377457]

35. Zhang DY, Chen SX, Yin P. Nat Chem. 2012; 4:208. [PubMed: 22354435]

36. Yin P, Choi HMT, Calvert CR, Pierce NA. Nature. 2008; 451:318. [PubMed: 18202654]

37. Li BL, Ellington AD, Chen X. Nucleic Acids Res. 2011; 39:e110. [PubMed: 21693555]

38. Zhang DY, Turberfield AJ, Yurke B, Winfree E. Science. 2007; 318:1121. [PubMed: 18006742]

39. Zhang DY, Seelig G. Nat Chem. 2011; 3:103. [PubMed: 21258382]

40. Zhang DY, Winfree E. J Am Chem Soc. 2009; 131:17303. [PubMed: 19894722] 
41. Baaske M, Vollmer F. ChemPhysChem. 2012; 13:427. [PubMed: 22213654]

42. Arnold S, Khoshsima M, Teraoka I, Holler S, Vollmer F. Opt Lett. 2003; 28:272. [PubMed: 12653369]

43. Zhang DY, Winfree E. Nucleic Acids Res. 2010; 38:4182. [PubMed: 20194118]

44. Suter JD, Howard DJ, Shi H, Caldwell CW, Fan X. Biosens Bioelectron. 2010; 26:1016. [PubMed: 20846848]

45. Lee M, Walt DR. Anal Biochem. 2000; 282:142. [PubMed: 10860511]

46. Zuker M. Nucleic Acids Res. 2003; 31:3406. [PubMed: 12824337] 

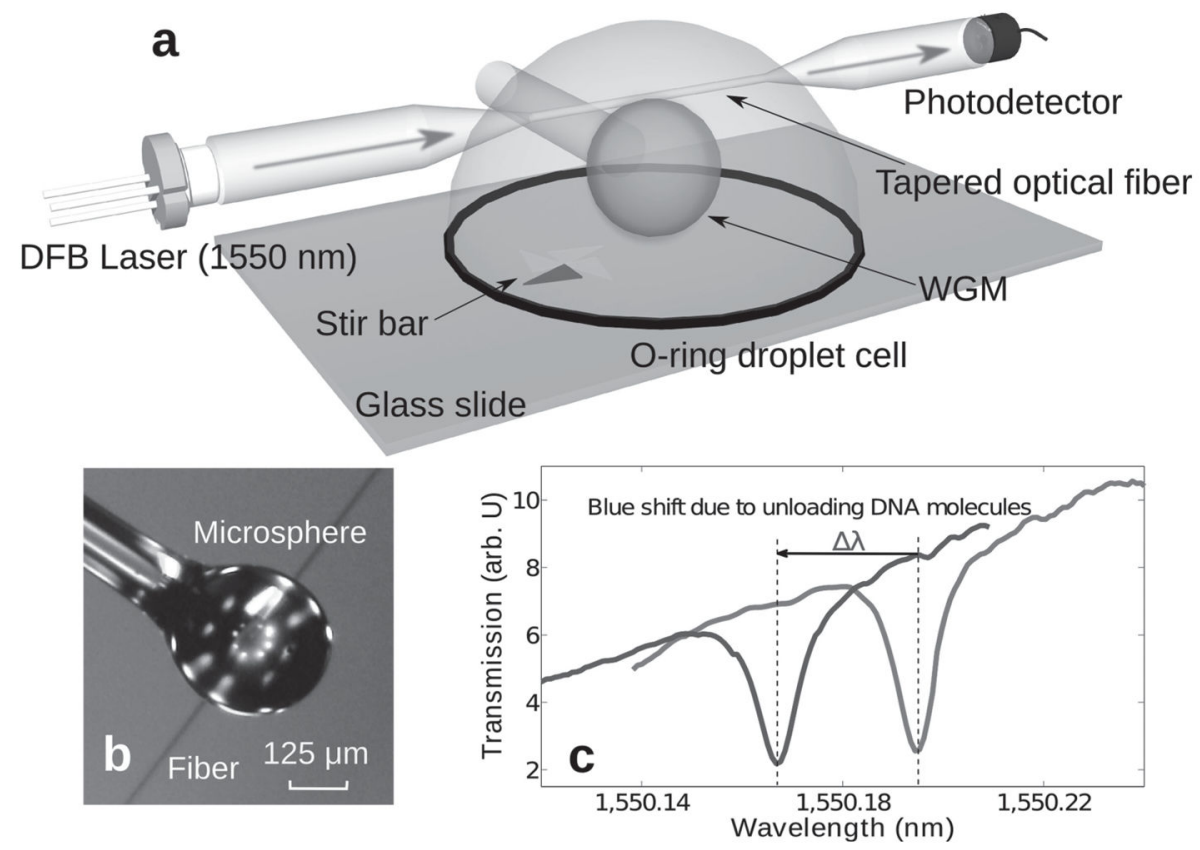

Figure 1.

Experimental setup for WGM biosensor. (a) Light from a DFB laser $(1550 \mathrm{~nm})$ is coupled into and out of a WGM microsphere resonator through a tapered optical fiber. The droplet sample cell is prepared from an o-ring glued on a glass slide. A miniature magnetic stir bar homogenizes the reaction. WGM transmission spectra are acquired by the photodiode in real time while rapidly sweeping the DFB laser wavelength by $\sim 0.2 \mathrm{~nm}$. (b) A micrograph of a glass microsphere (diameter $\sim 300 \mu \mathrm{m}$ ) coupled to a tapered optical fiber. (c) A typical spectrum of the microsphere, here before (red) and after (blue) the unloading of DNA from the surface of the microsphere. 
a

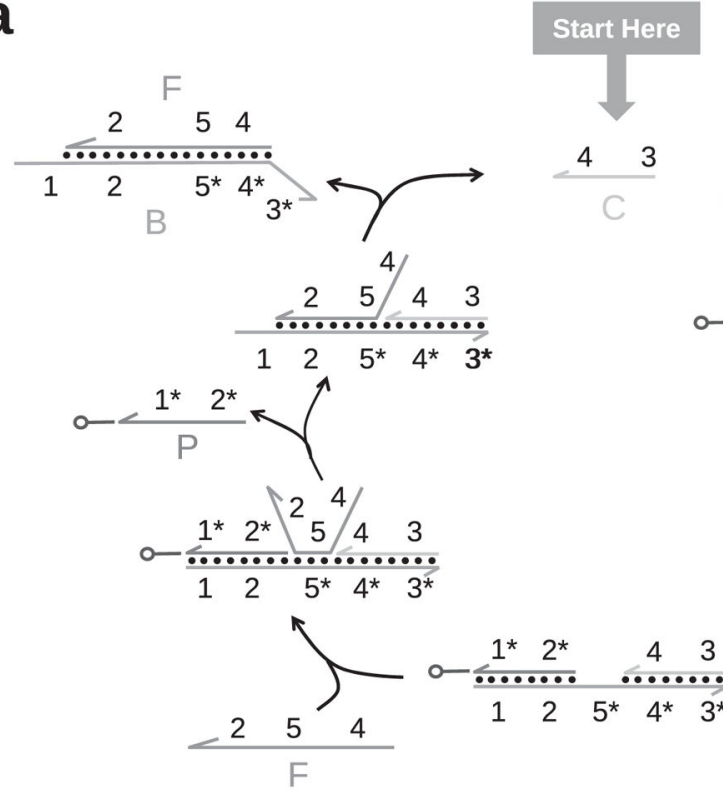

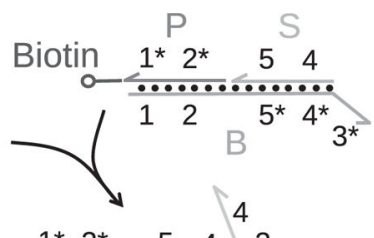

$1^{*} 2^{*} \quad 5 \quad 4 \quad 3$

0

$125^{*} 4^{*} 3^{*}$
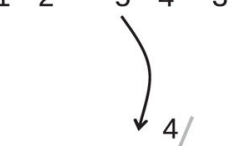

$1^{*} 2^{\star} 5 \quad 4 \quad 3$

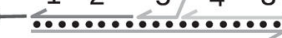

$125^{\star} 4^{\star} 3^{\star}$

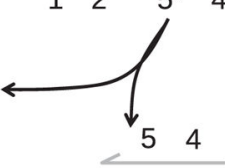

b

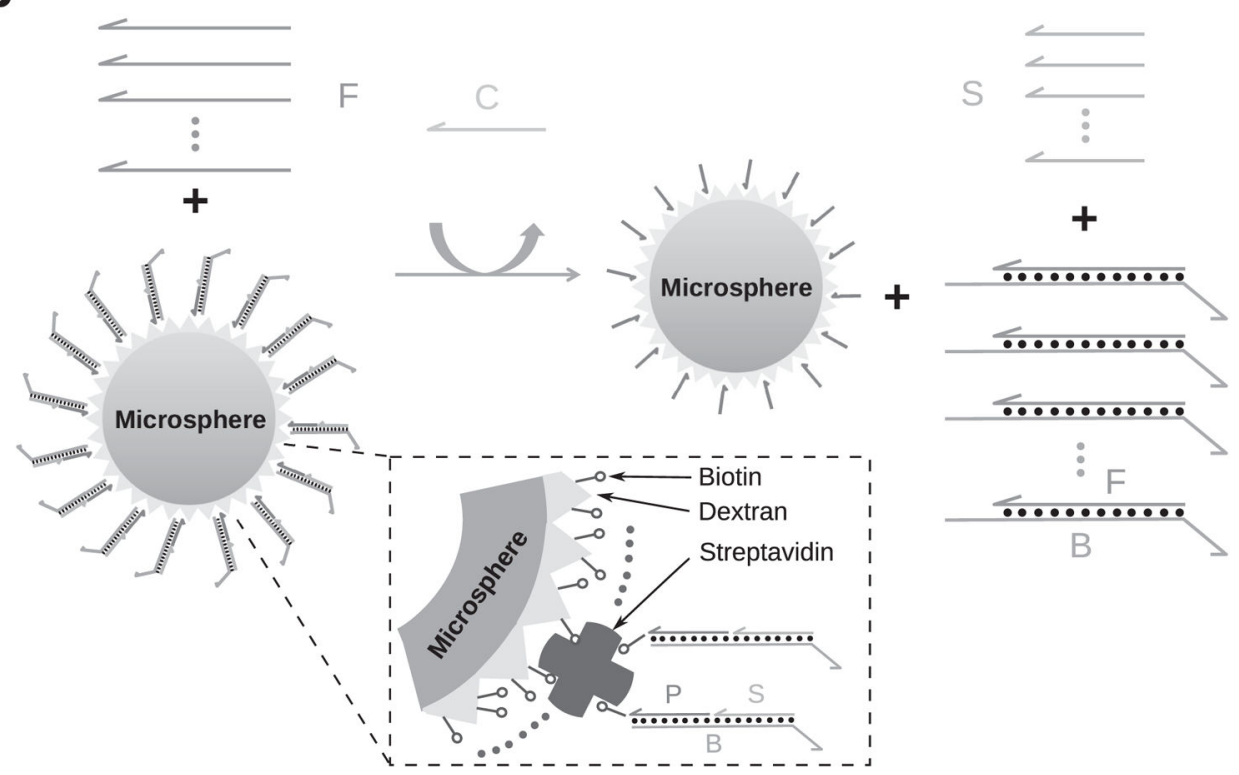

Figure 2.

The WGM biosensor integrated with the DNA catalytic network. (a) The designed catalytic pathway. In the absence of detection target $\mathrm{C}$, precursors SBP and $\mathrm{F}$ are metastable and do not interact significantly with each other. Target $C$ acts catalytically to enable the reaction between SBP and F, resulting in the release of S and B from P. Domains (displayed as numbers) represent continuous subsequences of nucleic acids that act as a unit in hybridization and dissociation; starred domains are complementary to non-starred domains. Sequences are shown in Table 2. (b) Catalysis causes mass unloading from the microsphere sensor surface. The $\mathrm{P}$ molecules are attached to the WGM microsphere via a biotinstreptavidin interaction; through the course of the catalytic reaction, S and B are unloaded 
from the surface. (b, inset) Schematic of DNA molecules attached to the sensor surface via biotin-streptavidin linkers (see Experimental Section). 

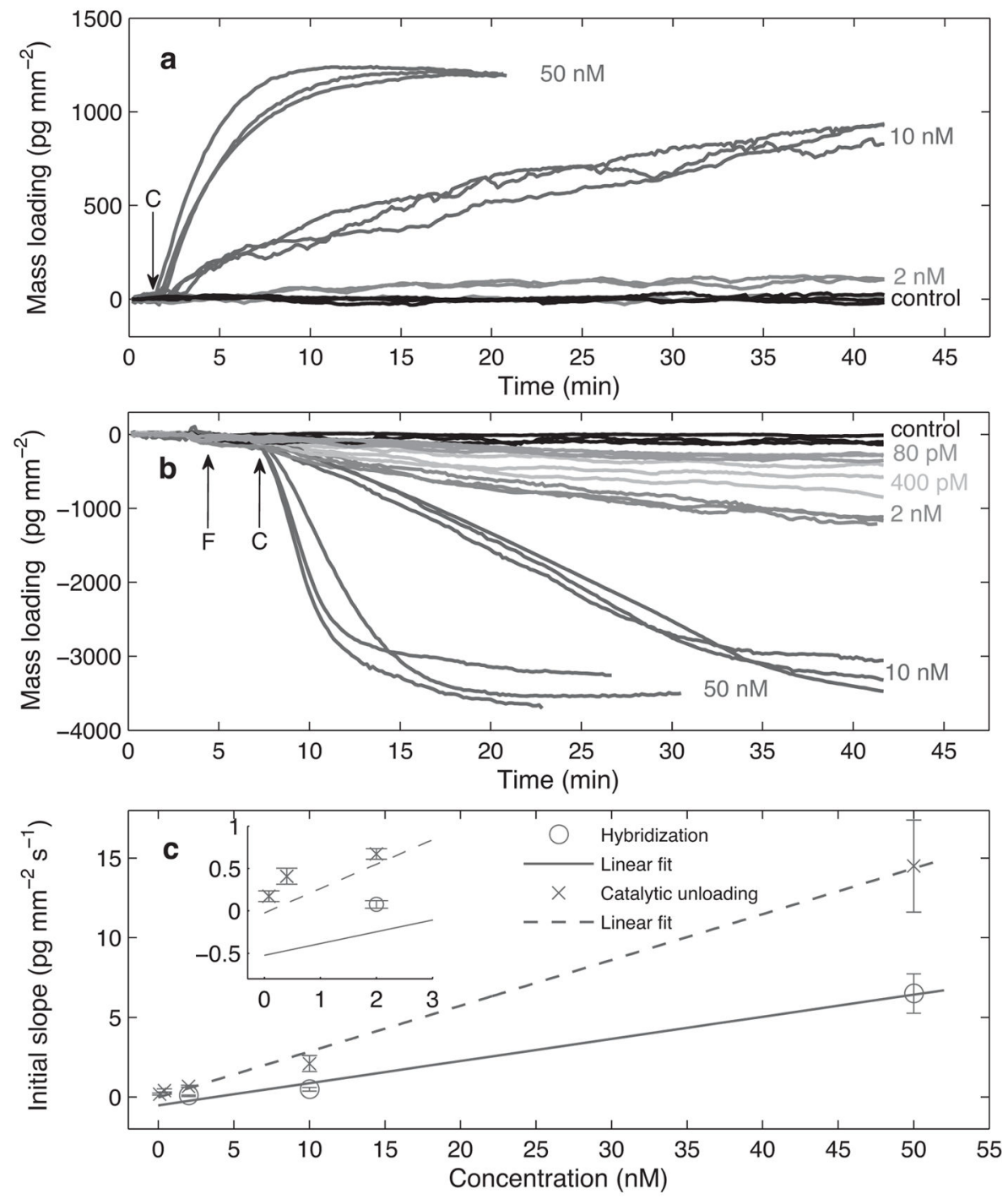

Figure 3.

Comparison of molecular sensitivity of the conventional WGM sensor versus the integrated strand displacement biosensor. (a) Direct hybridization of target $\mathrm{C}$ onto the probe $\mathrm{C}^{*}$ which was pre-attached to the microsphere surface. $4 \mu \mathrm{L}$ of target sequence $\mathrm{C}$ was injected at $\mathrm{t}=$ $200 \mathrm{~s}$ into the sample cell with $400 \mu \mathrm{L}$ buffer to achieve final C concentrations of $50 \mathrm{nM}$ (red), $10 \mathrm{nM}$ (blue) or $2 \mathrm{nM}$ (magenta). Control experiments (black) were done by immersing the sensors in the sample cell without injecting anything. (b) Integrated biosensor in which target $\mathrm{C}$ catalytically unloads strands $\mathrm{S}$ and B. $4 \mu \mathrm{L}$ of $\mathrm{F}$ was injected at $\mathrm{t}=200 \mathrm{~s}$ into the sample cell with $400 \mu \mathrm{L}$ buffer to achieve final $\mathrm{F}$ concentration of $400 \mathrm{nM}$. At $\mathrm{t}=$ $400 \mathrm{~s}, 4 \mu \mathrm{L}$ of $\mathrm{C}$ was injected to achieve final concentrations of $50 \mathrm{nM}$ (red), $10 \mathrm{nM}$ (blue) 2 $\mathrm{nM}$ (magenta), $400 \mathrm{pM}$ (green) or $80 \mathrm{pM}$ (orange). The control trace (black) shows the behavior of the system in the absence of C. (c) Averaged absolute initial slopes of mass loading/unloading for direct hybridization (red circle) and catalytic unloading (blue cross) as a function of concentration. The solid/dashed lines are the linear fit. (c, inset) A close-up for low concentrations. 


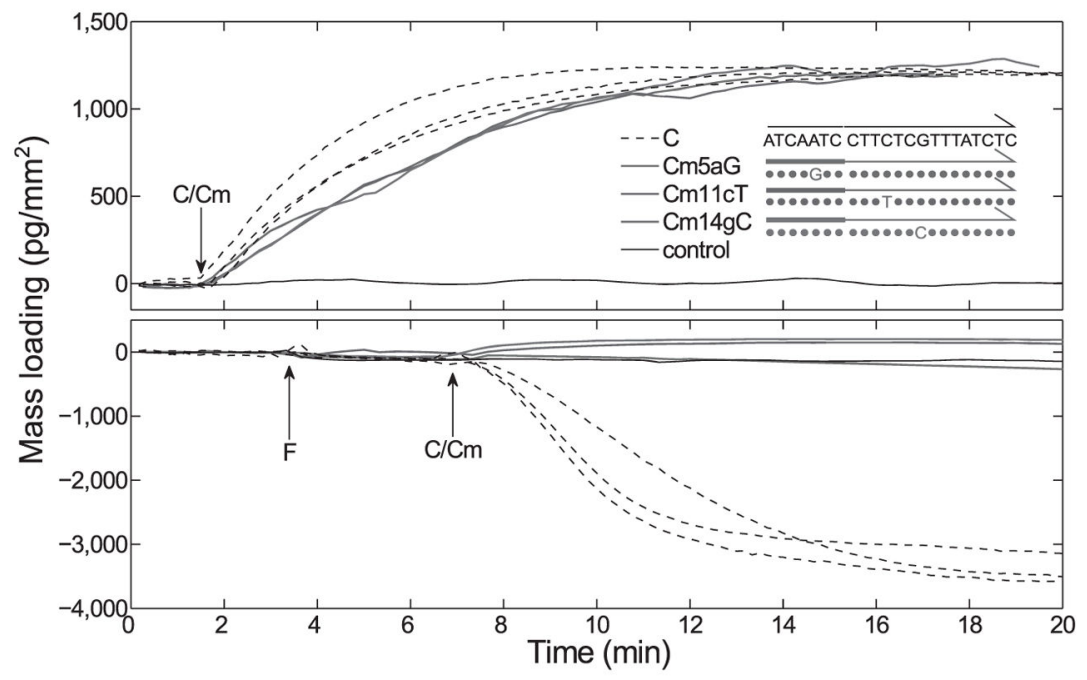

Figure 4.

Comparison of single nucleotide polymorphism (SNP) specificity of the conventional WGM biosensor versus the integrated biosensor. (a) SNP detection with the conventional hybridization method. The target (dashed lines) and three single nucleotide variants (red, blue, and green) were injected at $\mathrm{t}=100 \mathrm{sec}$ at $50 \mathrm{nM}$ concentration. The inset shows the sequences of the target and the single nucleotide variants; the thicker line segment denotes the toehold region. (b) SNP detection with the integrated biosensor. F with a final concentration of $400 \mathrm{nM}$ was injected at $\mathrm{t}=200 \mathrm{~s}$ and followed by the injection of target or single nucleotide variant ( $\mathrm{C}$ and $\mathrm{Cms}$, respectively) at $\mathrm{t}=400 \mathrm{~s}$ to a concentration of $50 \mathrm{nM}$. Colors of traces are consistent with those shown in panel (a). The initial slight increase in mass loading due to single nucleotide variants may be due to nonspecific interactions and/ or physical perturbation of system due to addition of reagents. 


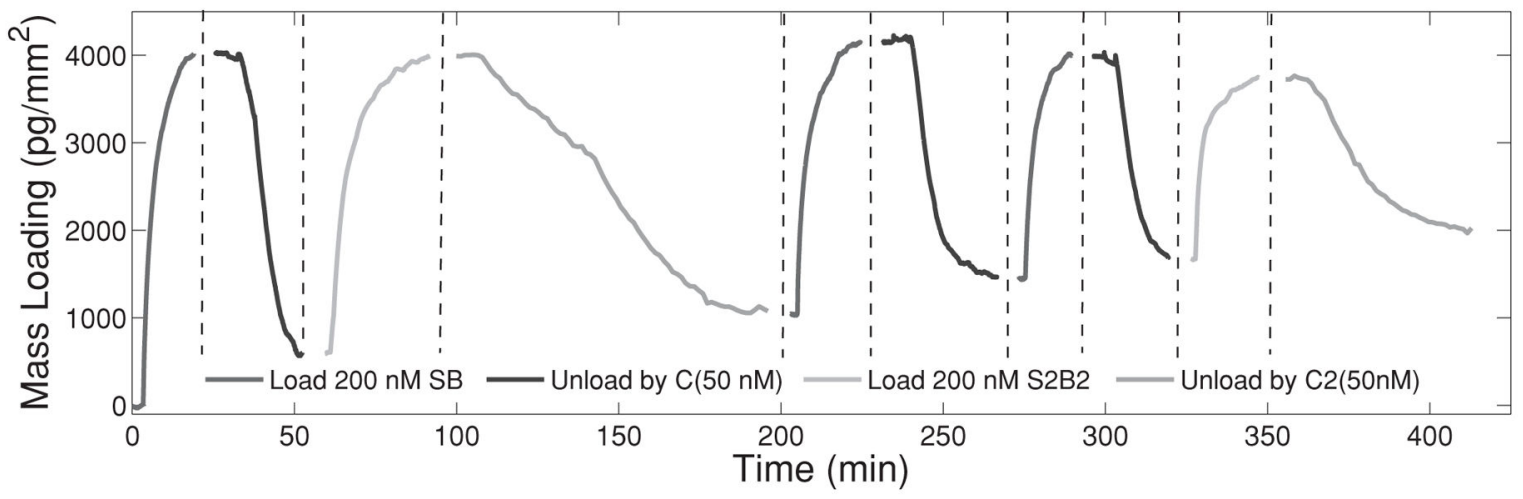

Figure 5.

Repeated reuse of integrated WGM biosensor to detect multiple different target sequences. Loading experiments were performed with $200 \mathrm{nM}$ of SB/S2B2 and unloading experiments were performed with $400 \mathrm{nM} \mathrm{F/F2}$ and $50 \mathrm{nM} \mathrm{C/C} 2$. The mass loadings were converted from the relative shifts of each loading/unloading cycle and offset so that the next loading/ unloading cycle starts where the last one ended. 


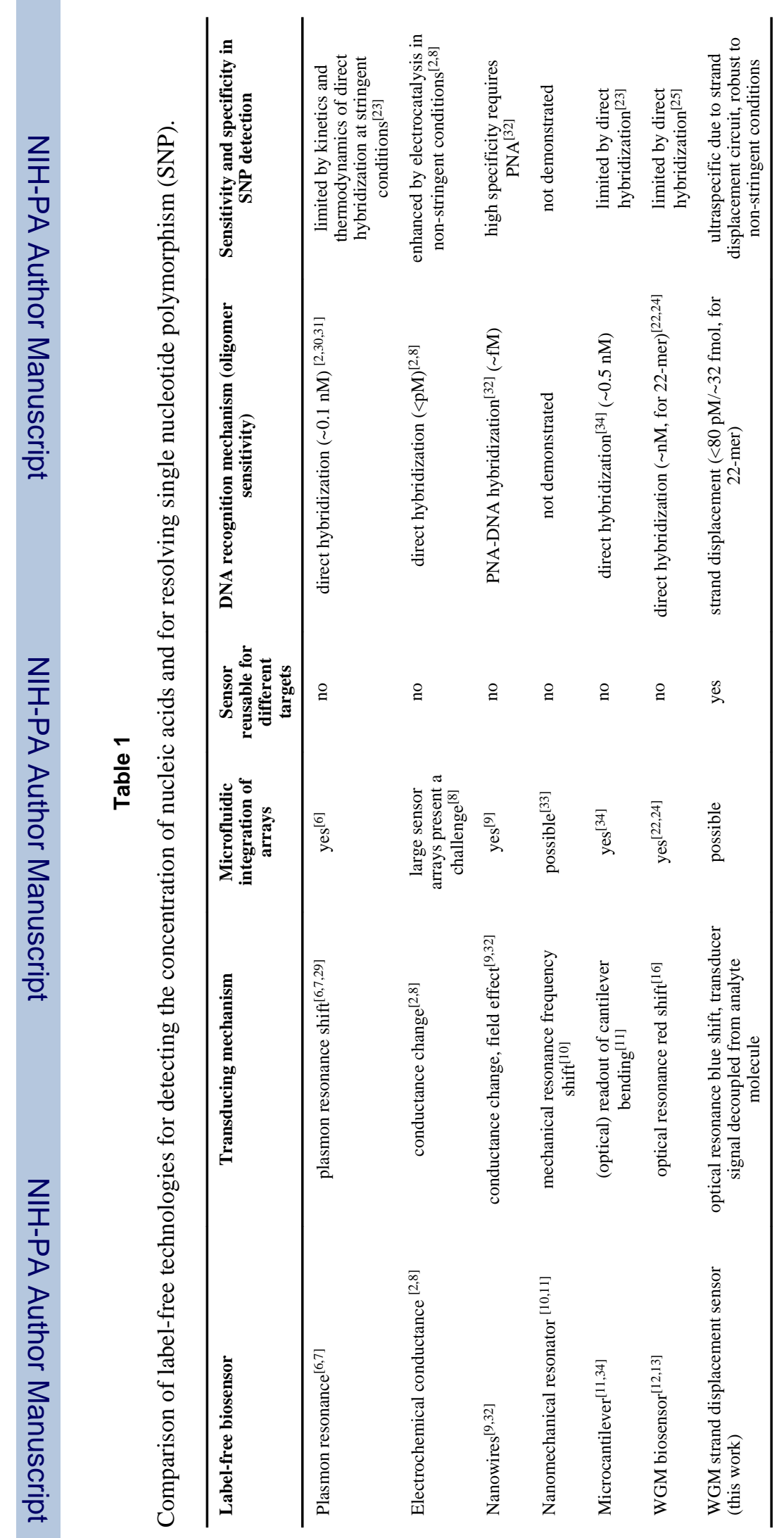

Small. Author manuscript; available in PMC 2014 July 14. 


\section{Table 2}

Sequences of the oligonucleotides.

\begin{tabular}{lrc}
\hline Domain & Sequences & Length (nt) \\
\hline $\mathrm{C}$ & 5'-ATCAATC CTTCTCGTTTATCTC-3' $^{\prime}$ & 22 \\
$\mathrm{C}^{*}$ & 5'-biotin-GAGATA AACGAGAAG GATTGAT-3' $^{\prime}$ & 22 \\
$\mathrm{~S}$ & 5'-CTTCTCGTTTATCTCCTGTA-3' $^{\prime}$ & 20 \\
$\mathrm{~B}$ & 5'-GCGATG GGTAAGAACTTTAGTG TACAG GAGATAAACGAGAAG GATTGAT-3' $^{\prime}$ & 49 \\
$\mathrm{~F}$ & 5'-CTTCTCGTTTATCTC CTGTA CACTAAAGTTCTTACC-3' $^{\prime}$ & 36 \\
$\mathrm{P}$ & 5'-CACTAAAGTTCTTACC CATCG-biotin-3' $^{\prime}$ & 21 \\
$\mathrm{Cm} 5 \mathrm{aG}$ & 5'-ATCAGTC CTTCTCGTTTATCTC-3' $^{\prime}$ & 22 \\
$\mathrm{Cm} 11 \mathrm{cT}$ & 5'-ATCAATC CTTTTCGTTTATCTC-3' $^{\prime}$ & 22 \\
$\mathrm{Cm} 14 \mathrm{gC}$ & 5'-ATCAATC CTTCTCCTTTATCTC-3' $^{\prime}$ & 22 \\
\hline
\end{tabular}

Small. Author manuscript; available in PMC 2014 July 14. 